\title{
VLBI FOR PROBING LARGE-SCALE MAGNETIC STRUCTURES OF STARS
}

\author{
J.-F. LESTRADE \\ Observatoire de Paris-Meudon, \\ CNRS-URA1757, F92195, Meudon, France
}

\begin{abstract}
By VLBI astrometry, we show that the two RSCVn type binaries, UX Ari and $\sigma^{2} \mathrm{CrB}$, have a preferred site of radio emission which is the intra-system region. It is known that radio emission from these stars is from the gyro-synchrotron process associated with large-scale magnetic fields. High gravity in the intra-system region might favor dense magnetic loops. Interactions in this region between loops attached to the surfaces of the two stellar components might produce reconnections required for electron acceleration.
\end{abstract}

\section{Introduction}

The structure and activity of the chromosphere and corona of the Sun are tied to its magnetic topology and this association should be even more direct for the enhanced fields of the rapidly rotating late-type stars, namely RS CVn's, T Tauri's, dMe's and Algol's. Doppler Imaging and Zeeman Doppler Imaging (see this volume) are techniques designed to reveal star spots and to measure directly the magnetic fields to improve our knowledge of this topology. Non-thermal radio emission from stars originates from regions of magnetic fields and high-angular VLBI observations of this emission is also a mean to probe indirectly this topology on large scale. Radio features a stellar radius in extent for a star at 30 parsecs can be resolved by VLBI. VLBI images of stars (UX Ari: Mutel et al. 1985; Cyg-X3: Molnar et al. 1988; LSI61303: Massi et al. 1993; HD283447: Phillips et al. 1996), are difficult to make because of the variability of their radio flux density but the flexibility of the newly commissioned VLBA should allow progress soon. VLBI visibility function studies (UX Ari: Massi et al. 1988, Algol: 
Lestrade et al. 1988) have contributed to show that during radio outbursts features smaller than a stellar diameter are present but that the size of the radio emitting region is typically 3 to 5 stellar radii during the postoutburst decay phase or during quiescence. VLBI astrometric observations of Algol have allowed to identify the corona of the late-type subgiant of this system, and not the B8 main sequence star, to be the site of the radio emission by tracking the position of the radio-emitting region over two orbital revolutions (Lestrade et al. 1993).

We present in this report two new series of VLBI astrometric observations of the RSCVn binaries, UX Ari and $\sigma^{2} \mathrm{CrB}$, that trace the location of their radio-emitting regions and we discuss implications for their magnetic topologies on large-scale.

\section{Very Long Baseline Interferometry}

Very Long Baseline Interferometry (VLBI) (Thompson et al. 1991) allows milliarcsecond angular resolution at radio wavelengths to measure directly high brightness temperature corresponding to non-thermal radio emission produced by relativistic electrons in magnetic fields. Typically, at centimeter wavelengths, the angular resolution over intercontinental baseline $B$ is $\theta \sim \frac{\lambda}{B} \sim 1$ milliarcsecond and brightness temperature in excess of $10^{8} \mathrm{~K}$ is measured. The radio emission mechanism in non-thermal radio stars usually invoked is the gyro-synchrotron process whose emission peaks at 10 to 100 times the gyro-frequency $(=2.8 \times T, \mathrm{MHz}$, Gauss $)$ and magnetic field strength $T$ deduced is between 30 and 300 Gauss for stars with $\mathrm{GHz}$ peaked spectrum (Dulk 1985).

VLBI is also an astrometric technique allowing submilliarcsecond precison at centimeter wavelengths. We have conducted several sessions of observations on UX Ari and $\sigma^{2} \mathrm{CrB}$ with VLBI arrays run under the auspices of the US Network, European Network, Deep Space Network and VLBA. The radio stars are weak radio sources compared to quasars usually observed by VLBI and we had to resort to the phase-referencing technique that allows long integration period of several hours and high-precision astrometry by the use of the most precise VLBI observable : the phase. A typical VLBI observation session for UX Ari and $\sigma^{2} \mathrm{CrB}$ was 5 to 10 hours with an array of 4 to 7 antennas located in the US and in Europe. The raw data were processed mainly at Haystack Observatory (Mass) and some at the Max Planck Institute fur Radioastronomie (Germany). The VLBI visibilities were inverted into phase-referenced maps for astrometry with the software SPRINT according the method described in detail in Lestrade et al. 1990. 


\section{VLBI observations of UX Arietis}

UX Ari is an RS CVn type close binary (G2V/K0IV) with a circular orbit which has a period of 6.4 days, an inclination of $60^{\circ}$ and a component separation of 3.4 milliarcseconds at the distance of 50 parsecs making it the largest binary in angular size of the active radio RS CVn's. UX Arietis is chromospherically active $(\mathrm{Ca} \mathrm{H}$ and $\mathrm{K})$ and is detected at X-ray and radio wavelengths. We have observed UX Ari by VLBI in the astrometric mode of phase-referencing at 10 epochs between 1983 and 1994. These VLBI data are used here to study in time the sky-projected location of the radio-emitting region, precisely, of the radio center of this region. Since the VLBI observations encompass such a long period and that there are no proper motion and trigonometric parallax accurate enough to model the main motion of the star projected on the sky, the VLBI positions of UX Ari measured at the 10 epochs were first used to solve for these astrometric parameters. Their VLBI precision is considerably higher than present optical determination and they will be used to link the future Hipparcos optical astrometric catalogue to the stable VLBI extragalactic frame of reference. However, the relevant part of this work here is the analysis of the VLBI post-fit position residuals of the astrometric adjustment. These astrometric residuals are displayed on the sky in Figure 1. The "zero residual point" shown as a diamond mark in Figure 1 is the barycenter resulting from the Least-Squares-fit that requires that the mean of the residuals be zero. Note that with the 10 epochs of observations and the relatively uniform coverage of the orbit (see Table 1), it is expected that the location of this zero point will not significantly change when more epochs of observations are added. These residuals have an rms of 0.8 milliarcseconds that is significantly larger than the VLBI measurement uncertainty that is estimated to be less than 0.3 mas in both right ascension and declination including noise and systematic errors caused by troposphere, ionosphere and geometry of the VLBI arrays used. It is noticeable that the residuals plotted in Figure 1 are distributed predominantly in the East-West direction in filling an area that matches the projected orbit based on the spectroscopic orbital elements of the binary system when the node, unknown to spectroscopy, is set to P.A. $=240^{\circ}$ and the center of the elliptic orbit is made to coincide with the location of the VLBI zero residual point. Although such a registration of the binary optical components upon the radio coordinate grid cannot be made $a$ priori because the optical and radio coordinate systems do not coincide at better than 50 milliarcseconds in this pre-Hipparcos catalog era, the match between the distribution of the residuals on the sky and the area within the projected spectroscopic orbit is striking. Physically, Figure 1 indicates that the center of the radio emission of UX Arietis wanders within 
TABLE 1. VLBI observations of UX Arietis

\begin{tabular}{lccr}
\hline Date & $\begin{array}{c}\text { Orbital } \\
\text { Phase (cy) }\end{array}$ & $\begin{array}{c}\text { Frequency } \\
(\mathrm{GHz})\end{array}$ & $\begin{array}{r}\text { Flux density } \\
(\mathrm{mJy})\end{array}$ \\
\hline 830727 & 0.212 & 5.0 & 150 \\
880307 & 0.988 & 5.0 & 14 \\
901116 & 0.859 & 5.0 & 5 \\
910612 & 0.135 & 5.0 & 40 \\
910919 & 0.448 & 5.0 & 96 \\
910921 & 0.758 & 5.0 & 59 \\
910922 & 0.914 & 5.0 & 56 \\
920115 & 0.777 & 8.4 & 14 \\
920322 & 0.287 & 8.4 & 330 \\
940528 & 0.059 & 8.4 & 42 \\
\hline
\end{tabular}

the intra-system region.

It is interesting to discuss particularly the July 1983 epoch since UX Ari could be mapped by VLBI because its flux density was relatively strong and constant. The structure mapped shows an elongated halo about the separation of the binary and an unresolved core only $10 \%$ of the total flux density that make the radio center very close to the center of the halo. It is noticeable that for this epoch the astrometric residuals in right ascension and declination are almost zero making the radio halo well centered on the the spectroscopic orbit registered as above. Note also that the halo is elongated predominantly in the East-West direction as the projected spectroscopic orbit. Differently, on 1992 September 19, 21, 22, during a phase of slow decay of the flux density of UX Ari, VLBI maps were also made and show that the radio source is mainly unresolved with Gaussian brightness distribution source size comparable with the diameter of the subgiant of this system. For these epochs numbered 5,6,7 in Figure 1, the astrometric residuals are consistent with the radio source being close to the ellipse of the projected orbit, i.e. associated with one of the stellar component rather than the intra-system region. There is no way to identify which one of the two stellar components is active with our present registration and node ambiguity of $180^{\circ}$.

\section{VLBI observations of $\sigma^{2} \mathrm{CrB}$}

$\sigma^{2} \mathrm{CrB}$ is an RS CVn-type close binary (F6V/G0V) with a quasi-circular orbit which has a period of 1.1 day, an inclination of $28^{\circ}$ and a component separation of 1.2 milliarcsecond at the distance of 23 parsecs. $\sigma^{2} \mathrm{CrB}$ is 


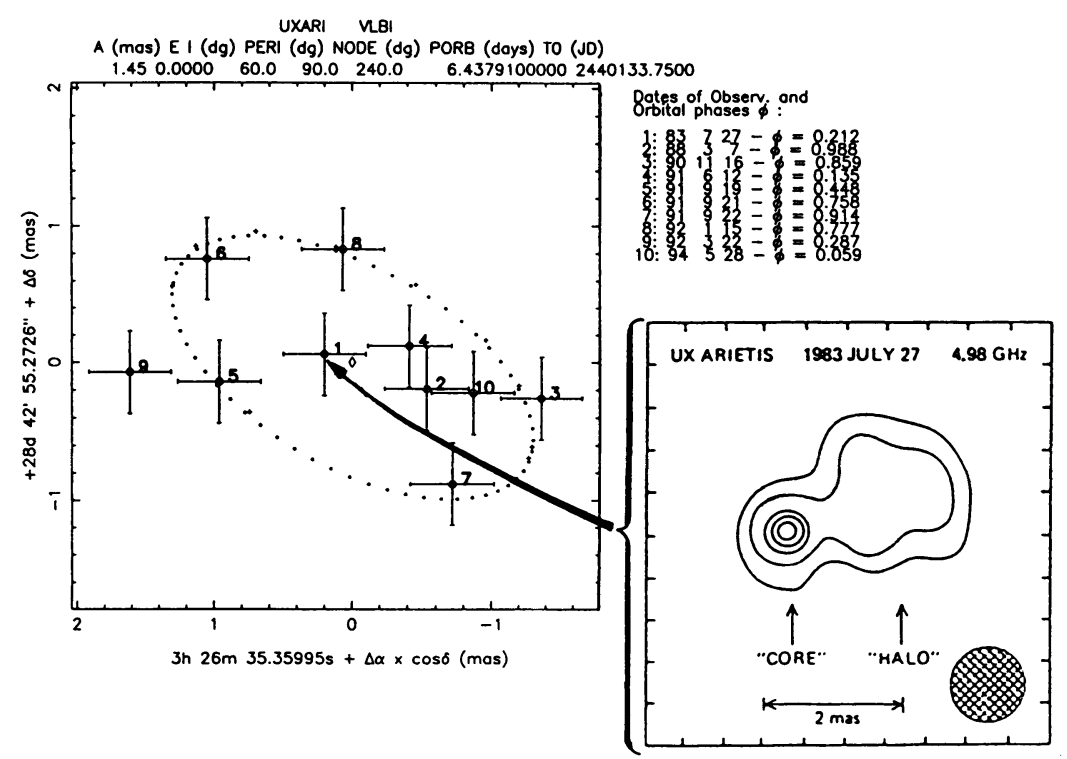

Figure 1. Sky distribution of the VLBI astrometric residuals numbered with epochs and the projected spectroscopic orbit (dots) of UX Arietis. The VLBI measurement uncertainties ( 0.3 milliarcsecond) in both coordinates are indicated by the cross in the bottom right corner. The radius of the subgiant in UX Arietis is 0.25 milliarcsecond. These astrometric residuals indicate that the center of the radio-emitting region of UX Arietis is wandering predominantly within the intra-system zone. According to our registration, the VLBI map of UX Ari on July 1983 is well centered on the theoretical projected orbit. Note that, as indicated, the angular scale of VLBI map is twice as small as the scale used to plot the astrometric residuals.

chromospherically active (CaII $\mathrm{H}$ and $\mathrm{K}$ ) and is detected at X-ray and radio wavelengths. We have observed $\sigma^{2} \mathrm{CrB}$ by VLBI in the astrometric mode of phase-referencing at 15 epochs between 1987 and 1994. Similarly to the analysis conducted for UX Ari, these VLBI data were used to solved for the astrometric parameters of the star and post-fit position residuals are here discussed in an attempt to locate the center of the radio emitting region in the system. These post-fit astrometric residuals are plotted on the sky along with the spectroscopic orbit in Figure 2. The node of this orbit is set to P.A. $=-40^{\circ}$ and its center is made to coincide with the zero residual point according to the procedure described for UX Ari above. It is interesting that the post-fit residuals for $\sigma^{2} \mathrm{CrB}$ are exactly circumscribed by the projected spectroscopic orbit. The orbit inclination of $28^{\circ}$ of $\sigma^{2} \mathrm{CrB}$ being smaller than for UX Ari $\left(60^{\circ}\right)$ the projected orbit is quasi-circular and it is noticeable that the the distribution of the residuals do reflect this geometry. 
TABLE 2. VLBI observations of $\sigma^{2} \mathrm{CrB}$

\begin{tabular}{lcccr}
\hline Date & $\begin{array}{c}\text { Orbital } \\
\text { Phase (cy) }\end{array}$ & $\begin{array}{c}\text { Frequency } \\
(\mathrm{GHz})\end{array}$ & $\begin{array}{r}\text { Flux density } \\
(\mathrm{mJy})\end{array}$ \\
\hline $8705 \quad 26$ & 0.558 & 5.0 & 10 \\
$8811 \quad 16$ & 0.768 & 5.0 & 28 \\
890413 & 0.287 & 5.0 & 7 \\
$9011 \quad 17$ & 0.369 & 5.0 & 4 \\
910412 & 0.988 & 5.0 & 19 \\
910914 & 0.648 & 5.0 & 4 \\
$9201 \quad 15$ & 0.965 & 5.0 & 5 \\
920405 & 0.958 & 8.4 & 18 \\
920608 & 0.889 & 8.4 & 13 \\
920803 & 0.057 & 8.4 & 3.2 \\
$9303 \quad 11$ & 0.148 & 8.4 & 15 \\
931002 & 0.444 & 8.4 & 4.7 \\
940325 & 0.885 & 8.4 & 16 \\
940925 & 0.427 & 8.4 & 4 \\
941123 & 0.045 & 8.4 & \\
\hline
\end{tabular}

Another finding is that there is a strong correlation of the astrometric residuals with the flux density variations of $\sigma^{2} \mathrm{CrB}$. The residuals lie on the spectroscopic orbit when $\sigma^{2} \mathrm{CrB}$ undergoes outbursts, i.e. when its flux density is higher than $>10 \mathrm{mJy}$, but are closely clustered within the orbit when in "quiescence", with flux density $<10 \mathrm{mJy}$. Flux densities of $\sigma^{2} \mathrm{CrB}$ are reported in Table 2 for all epochs. Quantitatively, the post-fit residual rms is 0.32 milliarcsecond for all 15 epochs and 0.20 milliarcsecond for the 7 epochs of "quiescence". Although this latter rms is larger than the measurement uncertainties of 0.08 milliarcsecond for this star, it corresponds to only $1 / 6$ of the binary separation implying that the radio emitting region is located in the intra-system zone and has a jitter small compared to the overall size of the binary system. We have depicted this configuration in Figure 3.

\section{Concluding remarks}

Radio emission from RS CVn is from the gyro-synchrotron process associated with their large-scale magnetic fields. By VLBI astrometry, we have shown that the two RSCVn type binaries, UX Ari and $\sigma^{2} \mathrm{CrB}$, have a preferred site of radio emission which is the intra-system region. We conclude that the topology of the magnetic fields favors this region. This is reminis- 


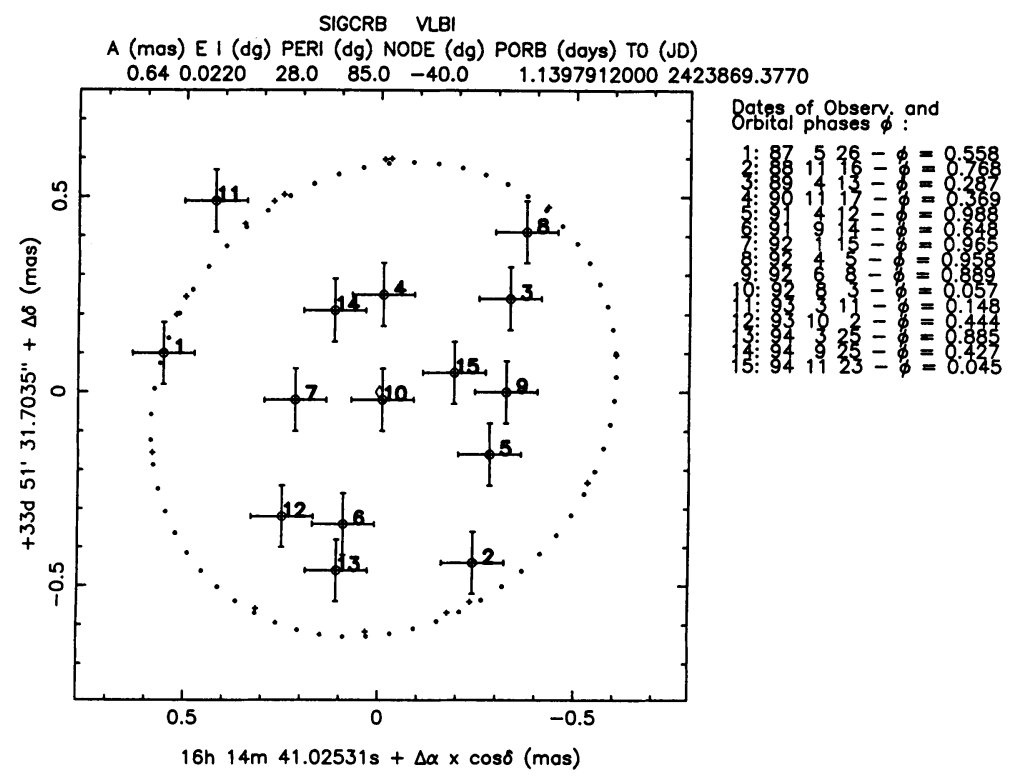

Figure 2. Sky distribution of the VLBI astrometric residuals numbered with epochs and the projected spectroscopic orbit (dots) of $\sigma^{2} \mathrm{CrB}$. The VLBI measurement uncertainties ( 0.08 milliarcsecond) in both coordinates are indicated by the cross in the bottom right corner. The radii of both stellar components in $\sigma^{2} \mathrm{CrB}$ are $\sim 0.21$ milliarcsecond. These astrometric residuals indicate that the center of the radio-emitting region of $\sigma^{2} \mathrm{CrB}$ is wandering predominantly within the intra-system zone.

cent of the concept presented by Uchida and Sakurai (1983) for RS CVn magnetic structure although detailed calculations have not been provided for this model. Nevertheless, our VLBI observations are consistent with the idea that there is more free energy available in the intra-system region of each of these two binaries. High gravity in this region might favor dense magnetic loops as suggested by van den Oord (these proceedings). Interaction in this region between loops attached to the surfaces of the two stellar components might produce reconnections required for electron acceleration.

We have attempted to associate more precisely the positions of the center of radio emission traced by the VLBI astrometric residuals of Figures 1 and 2 with predicted orbital phases of one of the two stellar components. We have found a good correlation for the epochs when $\sigma^{2} \mathrm{CrB}$ underwent outbursts but no correlation during quiescence and, generally, no correlation for UX Ari. These resultats are preliminary and need refinement. If confirmed, the site of radio outbursts in $\sigma^{2} \mathrm{CrB}$ could be one of the stellar components while low activity or quiescence emission would permeate the intra-system region. 


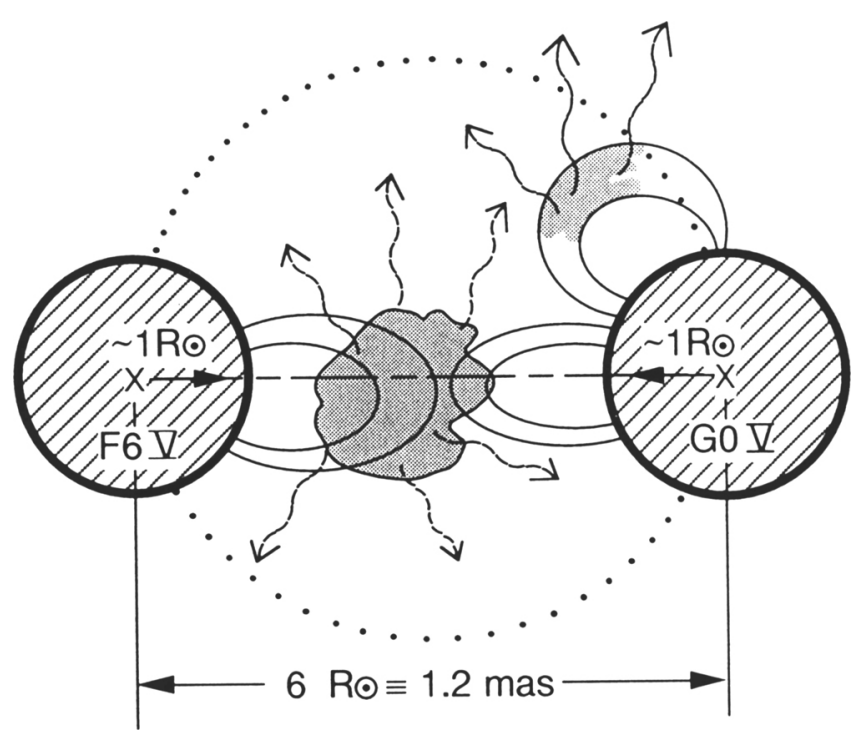

Figure 3. Sketch of the radio-emitting region in $\sigma^{2} \mathrm{CrB}$

Acknowledgements: I am grateful to R.B. Phillips, M.A. Titus at Haystack Observatory and to R.A. Preston, D.L. Jones at Jet Propulsion Laboratory for continous support all along the Hipparcos/VLBI link Project from which this study is derived.

\section{References}

Dulk G.A., 1985, ARA\&A 23, 169

Massi M., Felli M., Pallavicini R., Tofani G., Palagi F., Catarzi M., 1988, A\&A 197, 200 Massi M., Paredes J.M., Estalella R., Felli M., 1993, A\&A 269, 249

Molnar L.A., Reid M.J., Grindlay J.E., 1988, ApJ 331, 494

Mutel R.L., Lestrade J-F., Preston R.A., Phillips R.B., 1985, ApJ 289, 262

Lestrade J-F., Rogers A.E., Whitney A.E.E., Niell A.R., Phillips R.B., Preston R.A., 1990, AJ 99, 1663

Lestrade J.-F., Mutel R.L., Preston R.A., Phillips R.B., 1988, ApJ 328, 232

Lestrade J.-F., Phillips R.B., Hodges M.W., Preston R.A., 1993, ApJ 410, 808

Thompson A.R., Moran J.M., Swenson G.W., 1991, Interferometry and Synthesis in Radio Astronomy, Krieger Publ. Co., Malabar, Fl.

Uchida,Y., Sakurai,T., 1983, in P.B. Byrne and M. Rodono (eds) Activity in Red Dwarf Stars, Reidel (Dordrecht), p. 629.

Phillips, R.B. et al., 1996, A.J., (in press) 